\title{
Temporal dynamics of cerebro-cerebellar network recruitment during a cognitive task
}

\author{
S.H. Annabel Chen ${ }^{\mathrm{a}, \mathrm{c}}$, John E. Desmond ${ }^{\mathrm{a}, \mathrm{b}, *}$ \\ ${ }^{a}$ Department of Radiology, Stanford University School of Medicine, Lucas MRS Building, MC:5488, Stanford, CA 94305-5488, USA \\ ${ }^{\mathrm{b}}$ Neurosciences Program, Stanford University School of Medicine, Stanford, CA 94305, USA \\ ${ }^{\mathrm{c}}$ Department of Psychology, National Taiwan University, Taipei, Taiwan
}

Received 4 March 2004; received in revised form 14 December 2004; accepted 16 December 2004

Available online 25 February 2005

\begin{abstract}
Previous investigations have demonstrated that two regions in the right cerebellum, one located superiorly in hemispheral lobule VI/Crus I and another located inferiorly in hemispheral lobule VIIB/VIIIA, are activated during verbal working memory performance. On the basis of functional neuroimaging patterns of activation, as well as known cortico-pontine and ponto-cerebellar projections, the superior region has been hypothesized to contribute to the articulatory control system of working memory whereas the inferior region has been linked to the phonological store. The present study used event-related fMRI and individual estimates of hemodynamic response for both the cerebellum and neocortex to test this model and characterize the task phase specific cerebro-cerebellar activations for a Sternberg verbal working memory task. Results demonstrated that the right superior cerebellum showed the strongest activation during the initial encoding phase of the task, and, consistent with predictions, a similar pattern was observed in left opercular inferior frontal and premotor regions. In contrast, the right inferior cerebellum exhibited the greatest activation during the maintenance phase of the task, and as predicted, corresponded with activation in the left inferior parietal lobule. The significance of the results with respect to cerebro-cerebellar models of verbal working memory and to theoretical accounts of cerebellar involvement in cognition is discussed.
\end{abstract}

(C) 2005 Elsevier Ltd. All rights reserved.

Keywords: Cerebellum; Cognition; Event-related; Functional neuroimaging; Verbal working memory; Cerebro-cerebellar networks

In the last 15 years since cerebellar activation during cognitive performance was first revealed by functional neuroimaging (Petersen, Fox, Posner, \& Mintun, 1989) there has been growing interest in the contribution of cerebrocerebellar networks to cognitive function (Desmond \& Fiez, 1998; Marien, Engelborghs, Fabbro, \& De Deyn, 2001). In characterizing these networks, tasks that not only reliably elicit cerebellar and neocortical activations but also are capable of parametric manipulation and precise control of stimulus and response requirements offer significant advantages. One such task is the Sternberg (1966) verbal working memory paradigm, which has in addition to all of these characteristics a strong theoretical framework for interpreting brain activations.

\footnotetext{
* Corresponding author. Tel.: +1 650498 5368; fax: +1 6507235795

E-mail address: dr.jdesmond@gmail.com (J.E. Desmond).
}

In this framework (Baddeley, 1992, 1986) a phonological loop is hypothesized to permit the short-term maintenance of verbal information. This loop is assumed to be comprised of two components, a phonological store, which can hold speech-related information for 1-2 s, and an articulatory control system, which serves to subvocally refresh the contents of the phonological store. Neuroimaging studies have embraced this model, capitalizing on the wealth of behavioral studies supporting it, in order to identify neural substrates underlying these processes. These efforts have linked articulatory control processes to left inferior frontal regions and phonological storage to left inferior parietal regions (Awh et al., 1996; Fiez \& Raichle, 1997; Fiez et al., 1996; Jonides et al., 1998; Paulesu, Frith, \& Frackowiak, 1993; Smith \& Jonides, 1998).

Desmond, Gabrieli, Wagner, Ginier, and Glover (1997) similarly relied on Baddeley's model of articulatory control 
and phonological storage to interpret the patterns of cerebellar activation observed in verbal working memory. That study characterized the lobular distribution of two spatially and functionally distinct activations in the cerebellum, one that appeared bilaterally in the superior hemisphere (lobule VI/Crus I), and another that was located predominately in the right inferior hemisphere (lobule VIIB). Based on known patterns of cortico-pontine (Schmahmann, 1996) and pontocerebellar (Brodal, 1979) projections, they postulated that the superior and inferior cerebellar foci are similarly linked to articulatory control and phonological storage processes, respectively, via anatomical connections to frontal and parietal cortex. This interpretation, based on anatomical observations, was also supported by the pattern of functional activation results: the superior cerebellum activated not only during verbal working memory but also during a rehearsal control task that required repeated externally guided subvocal rehearsal of letters but no memory, thereby exhibiting a pattern of activation consistent with an articulatory control system. In contrast, the responses of the inferior cerebellum were more closely linked to processes dependent on phonological storage, exhibiting activation only during the working memory condition when internally guided rehearsal and phonological storage was required (Desmond, 2001a; Desmond et al., 1997). These observations were recently replicated and extended by Chen and Desmond (2005), who found that, as predicted by the model, both posterior frontal and superior cerebellar activations occurred during working memory and rehearsal control tasks, but that parietal and inferior cerebellar activations occurred exclusively for working memory.

The present study sought to further test the validity of this cerebro-cerebellar model of verbal working memory using event-related functional MRI (fMRI). The Sternberg task, with three temporally distinct phases, is ideally suited for event-related investigations. These phases consist of the initial encoding phase, in which two to six letters are briefly presented, the maintenance phase, in which the verbal information is kept in mind through subvocal rehearsal, and the retrieval phase, in which the stored information is utilized to make a response. Accordingly, subjects were scanned during performance of this task using a relatively high rate of fMRI data acquisition, and to insure maximum accuracy for characterizing phase specific responses, subject-specific estimates of hemodynamic response were obtained for both cerebellum and neocortex. We hypothesized that the phase(s) of the task to which the superior and inferior cerebellum responds would be informative as to the nature of cerebellar contributions to this cognitive function, as the three phases have different processing requirements. The encoding phase emphasizes the acquisition of information, the rapid conversion of visual to phonological code, and the rapid construction of an articulatory motor plan before the letters are removed from view. The maintenance phase emphasizes internally guided articulatory rehearsal to refresh the phonological store. The retrieval phase emphasizes the utilization of the maintained information and decisional processes. We further hypothesized that the cerebro-cerebellar regions responsive to each phase would be consistent with the anatomically based predictions of Desmond et al. (1997) such that phase-specific activations of superior cerebellum would be associated with concomitant activations in left frontal cortex and that activations in inferior cerebellum would be associated with concurrent activations in left parietal cortex.

The Sternberg task has been characterized as one in which encoding and maintenance of information is dominant, whereas alternative tasks such as n-back emphasize manipulation of information (Smith \& Jonides, 1999; Smith, Jonides, Marshuetz, \& Koeppe, 1998; Veltman, Rombouts, $\&$ Dolan, 2003). The task phase(s) during which activations occur in the present study will therefore help to clarify the relative contributions of different cerebro-cerebellar netwoks to encoding and maintenance processes. For example, in the block design study of Chen and Desmond (2005), common activations in frontal and superior cerebellar regions were observed for a Sternberg verbal working memory task and for a rehearsal control task. These tasks had similar requirements during the maintenance phase in that both tasks had covert articulation during that phase. Thus, one could argue that the common frontal-superior cerebellar activations observed in that study likely occurred during the maintenance phase. On the other hand, both tasks also had similar requirements for rapidly converting the externally (visually) presented letters into an articulatory motor pattern; on this basis one could also argue that the commonalities in activations were likely due to the encoding phase. Because the cerebellum has been shown to preferentially respond to rapid articulatory requirements (Wildgruber, Ackermann, \& Grodd, 2001), and because of the necessity for rapid articulatory preparation during the encoding phase of the task, we predict that frontal and superior cerebellar activations will be observed during the encoding phase. In contrast, parietal and inferior cerebellar activations are predicted to occur during the maintenance phase, reflecting repeated refreshment of the phonological store.

The results of Chen and Desmond (2005) revealed that frontal lobe activation common to working memory and rehearsal control tasks occurred in more posterior frontal regions (BA 6/44), whereas more anterior frontal regions were activated in the working memory task but not the rehearsal control task. We hypothesize that the posterior activations reflected the rapid initial construction of the articulatory trajectory under external guidance that occurred during stimulus encoding. These posterior regions are therefore predicted to activate in conjunction with superior cerebellum during the encoding phase in the present study. In contrast, we hypothesize that the more anterior frontal regions that were specific to the working memory task represent internally guided articulatory processes, and these regions are predicted to activate during the maintenance period. 


\section{Methods}

\subsection{Participants}

Fifteen right-handed ( 7 females, 8 males) native Englishspeaking young-adults, with no diagnosed psychological or neurological illness, were recruited from the university community. The study was approved by Stanford University Institutional Review Board and granting agency, and was performed in accordance with the ethical standards laid down in the 1964 Declaration of Helsinki. All participants gave their informed consent prior to their inclusion in the study. Age ranged from 18 to 28 years with a mean of 22.53 years (S.D. $=2.7$ years) and mean years of education was 17.27 years $($ S.D. $=3.45$ years $)$ with a range of 13-24 years.

\subsection{Task description}

Participants were presented with an array of either two or six letters for $2 \mathrm{~s}$, and were given either 4 or $6 \mathrm{~s}$ to rehearse during the maintenance phase. They were instructed to rehearse the letters subvocally in a sequential order. A probe letter was presented during retrieval for $1 \mathrm{~s}$, and participants indicated within $3 \mathrm{~s}$ if the letter matched the array by pressing the button under the index finger for "match" and the button under the third finger for "not-match" with their right hand (see Fig. 1a). Trials were jittered with an inter-trial interval ranging from 5 to $8 \mathrm{~s}$, and 16 blank trials that did not have the retrieval phase were included in each session. All subjects performed 5 sessions of 48 ( 24 per condition) trials each. Each session lasted between 641 and $655 \mathrm{~s}$. The presentation of conditions was randomized within each session and the order of session was randomized for each subject. To obtain an individualized hemodynamic response functions (HRFs) for each subject, a finger tapping task (see Fig. 1b), consisting of a button press with the right index finger every 29-31 s for $10 \mathrm{~min}$, was also performed. This task reliably elicits activation from the left motor cortex, and right (ipsilateral) anterior superior cerebellar cortex (Desmond et al., 1997) (see Fig. 1b). Stimuli were created with and driven by Psyscope V1.2.5 (Cohen, MacWhinney, Flatt, \& Provost, 1993) (http://psyscope.psy.cmu.edu) presented on an Apple Macintosh Power PC and displayed visually with a magnetcompatible projector. Prior to testing in the fMRI environment, subjects practiced 20 trials of each of the 2 load conditions to become familiarized with the task, using their right hand for responding.

\subsection{MRI data acquisition}

All MRI data were acquired on a GE 3T scanner equipped with a gradient echo spiral pulse sequence using a quadrature endcap birdcage resonator head coil. Structural MRI protocols consisted of a SpinEcho localizer scan and two T2weighted Fast SpinEcho (FSE) anatomical scans (oblique axial acquisition; TE $=85 \mathrm{~ms} ; \mathrm{TR}=3000 \mathrm{~ms} ; \mathrm{FOV}=24 \mathrm{~cm}$; $256 \times 192$ matrix; $\mathrm{NEX}=1.0$; slice thickness $=5 \mathrm{~mm} ; 1$ in-

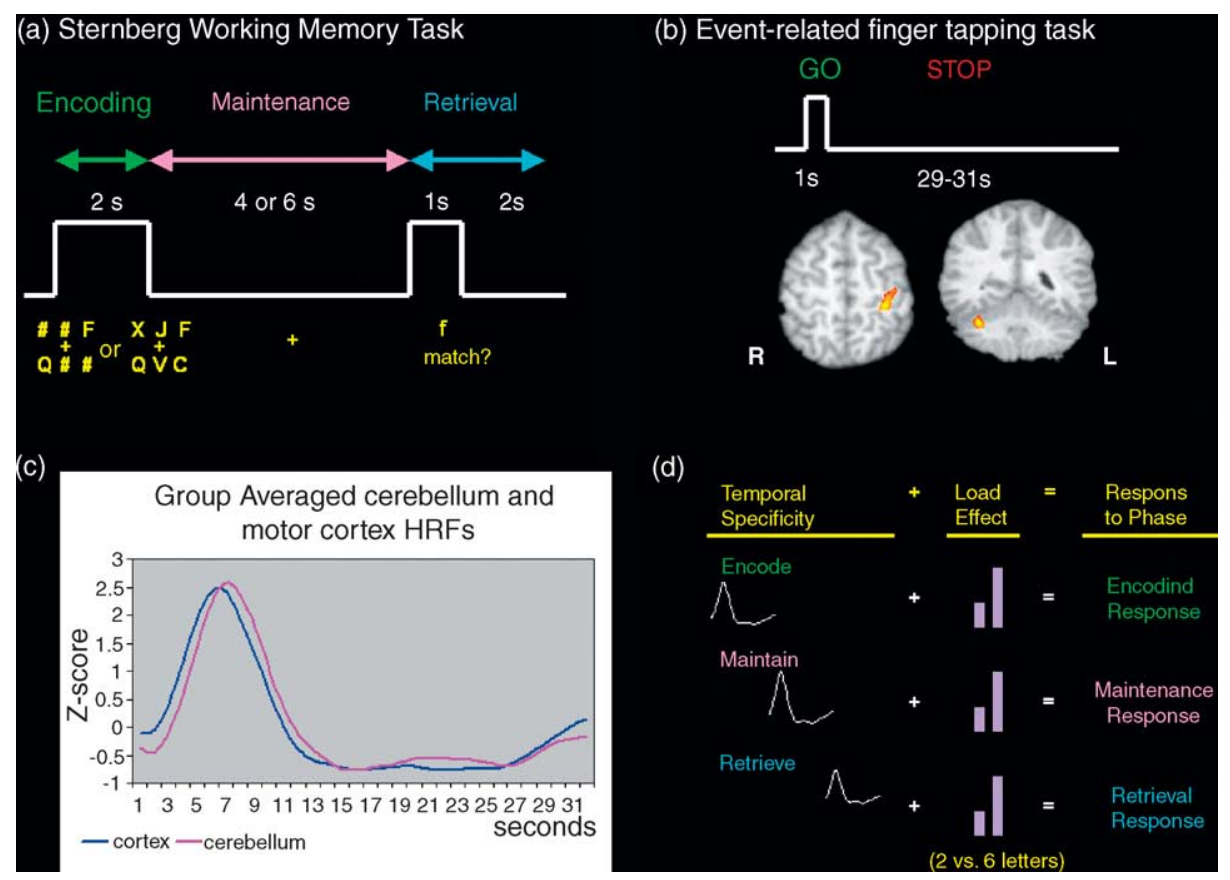

Fig. 1. (a) Event timing for a single trial of the Sternberg verbal working memory task; (b) event-related finger tapping task used to obtain subject-specific cortical and cerebellar hemodynamic response functions (HRFs); (c) averaged HRFs from the left motor cortex and right superior cerebellum resulting from the finger tapping task, illustrating that, on average, there were subtle timing differences in cerebellar and motor cortical HRFs; (d) summary of conjunction procedure used for classifying activations. Each voxel designated as responsive to encoding, maintenance, or retrieval phases were required to exhibit both temporal specificity and sensitivity to memory load. 
terleave) used for spatially registering the fMRI data. The T2-weighted FSE anatomical scan (whole brain FSE) covered the whole brain with 16 oblique slices of $7 \mathrm{~mm}$ thickness acquired in an oblique axial plane, rotated $25^{\circ}$ with respect to the AC-PC line. This angle optimized acquisition of both cerebellar and neocortical structures. fMRI data were collected last based on the cerebellum FSE prescription and acquired with a $\mathrm{T}^{*}{ }^{*}$-weighted gradient echo spiral-in/out (Glover \& Law, 2001) pulse sequence $\left(\mathrm{TE}=30 \mathrm{~ms} ; \mathrm{TR}=1000 \mathrm{~ms}\right.$; flip $=61^{\circ}$; inplane resolution $=3.75 \mathrm{~mm}$; slice thickness $=6 \mathrm{~mm}$ skip $1 \mathrm{~mm} ; 16$ oblique-axial slices; FOV $=24 \mathrm{~cm} ; 1 \mathrm{NEX).} \mathrm{For} \mathrm{display} \mathrm{pur-}$ poses, group activations were superimposed onto a normalized T1-weighted spoiled grass (SPGR) image. Scanner data collection was synchronized with the behavioral task via interfacing with the Psyscope software. The examiner spoke to the participants via the scanner's intercom system prior to each scan to review the test instructions and announce that the test was about to begin.

\subsection{Functional data analysis}

The SPM99 software package (Wellcome Department of Cognitive Neurology) was used for preprocessing and statistical computations. As described above, participant-specific HRFs were obtained from the left motor cortex and right superior cerebellum, respectively, using an event-related finger tapping task. To estimate the HRF, a Fourier set of four (sine/cosine) basis functions was used to model finger tap responses in a $32 \mathrm{~s}$ window. The average resulting response from the basis set was then calculated from the region of interest with maximum response in the right anterior superior cerebellar hemisphere and the left motor cortex. The HRFs obtained from each participant were convolved with reference waveforms for the encoding ( $2 \mathrm{~s})$, maintenance ( 4 or $6 \mathrm{~s}$ ), and retrieval $(3 \mathrm{~s})$ events in order to create customized neocortical and cerebellar regressors for each participant. Simulations of voxels responding to various combinations of encoding, maintenance, and retrieval events verified that this customized regressor approach correctly distinguished voxel phase responses.

Standard image preprocessing steps were performed, including slice timing correction, motion correction, anatomical coregistration, normalization to Montreal Neurological Institute (MNI) stereotaxic space, and spatial smoothing ( $\mathrm{FWHM}=5 \mathrm{~mm}$ ). Individual statistical maps were computed for each subject using the general linear model approach as implemented in SPM99, with high pass filtering of $34 \mathrm{~s}$ and no global scaling. A random effects analysis was then performed to make inferentially valid maps of average responses to encoding, maintenance, and retrieval phases of the task. This analysis was performed by computing one contrast volume per subject and using these volumes to calculate one-sample $t$-test values at every voxel.

In order to classify a voxel as responsive to a specific phase of the task, i.e., encoding, maintenance or retrieval, we re- quired that it showed both, temporal specificity for that phase and a load effect for the two versus six letters. Conjunctions of the phase contrast (which identified voxels significantly responding to the encoding, maintenance, and/or retrieval regressors pooled over high and low load conditions) with the load contrast (which identified voxels showing a significant high load minus low load subtraction for a given phase) were used to identify these voxels (see Fig. 1d). An initial $p<.05$ threshold was used in the conjunction to identify phase and load sensitive voxels; the resulting set of voxels were then thresholded at $p<.001$ according to the $\operatorname{SPM}\{Z\}$ values for the phase contrast. Voxels could be responsive to more than one phase of the task, and thus the depictions of encoding, maintenance, and retrieval related activations in Fig. 2 do not imply mutually exclusive voxel sets. Custom-written software was used to display the activation maps coregistered on the normalized TI-weighted SPGR anatomy (Desmond \& Lim, 1997).

The stereotaxic atlas of Talairach and Tournoux (1988) was used for identifying activated regions and reporting activation coordinates. Activations in the cerebellum were characterized using the atlas of Schmahmann, Doyon, Toga, Petrides, and Evans (2000). Coordinates depicted in Tables 1 and 2 were transformed from MNI into the Talairach coordinate system using the following transformations (Matthew Brett, http://www.mrccbu.cam.ac.uk/Imaging/mnispace.html). For MNI coordinates superior to the anterior commissure-posterior commissure (AC-PC) line (i.e., $Z$ coordinate greater than or equal to 0 ): $X^{\prime}=0.9900 X, Y^{\prime}=0.9688 Y+0.0460 Z$, and $Z^{\prime}=-0.0485 Y+0.9189 Z$, where $X, Y, Z$ refer to the MNI coordinates and $X^{\prime}, Y^{\prime}, Z^{\prime}$ refer to the Talairach coordinates. For MNI coordinates below the AC-PC line (i.e., $Z<0$ ), the transformations were $X^{\prime}=0.9900 X, Y^{\prime}=0.9688 Y+0.0420 Z$, and $Z^{\prime}=-0.0485 Y+0.8390 Z$.

\section{Results}

Behavioral responses obtained during the scans showed a high accuracy rate (percentage of hits) at $94 \%$ for the low load (two letters) condition and $88.5 \%$ for the high load (six letters) condition $(F(1,14)=33.4, p<0.0001)$. The mean of the median reaction time for correct responses was faster for low load $(832 \mathrm{~ms})$ than high load $(1033 \mathrm{~ms})(F(1,14)=21.5$, $p<0.0004)$.

Results of the neuroimaging analyses are summarized in Table 1 and Fig. 2, which illustrate significant activations for the encoding, maintenance, and retrieval phases, averaged over 15 subjects. Significant voxels in the figure represent regions that exhibited both temporal specificity to the task phase and sensitivity to working memory load, using a conjunction approach summarized in Fig. 1d. To insure that cerebellar and neocortical phase-dependent responses were identified as accurately as possible, the hemodynamic response function (HRF) for each subject was estimated for both the 


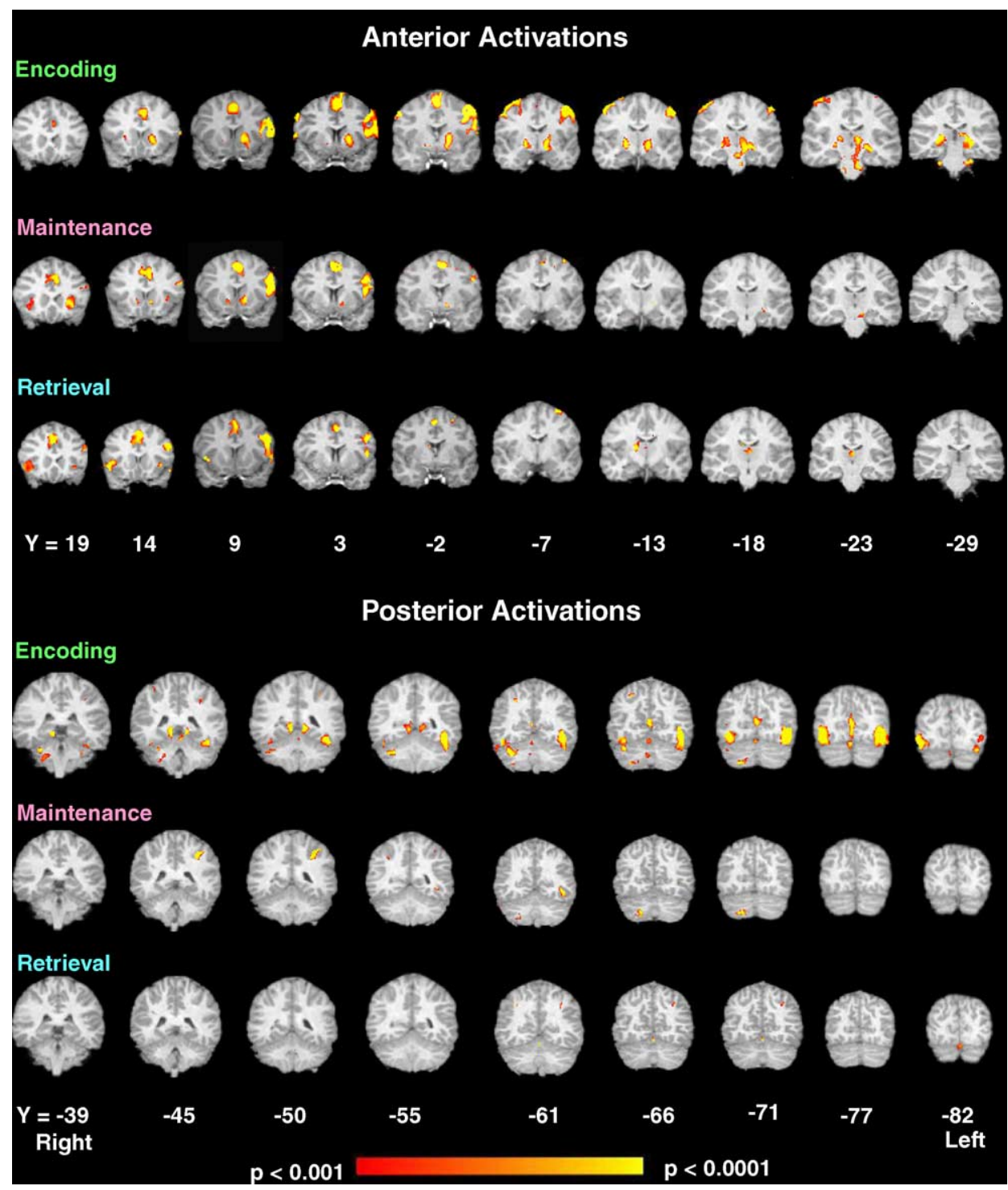

Fig. 2. Brain regions responsive to the encoding, maintenance and retrieval phases of the working memory task averaged over all subjects. Voxels depicted in the red-yellow color scale were computed using conjunctions of load and task phase contrasts and thus, represent regions that are both responsive to the specific phase of the task and sensitive to working memory load. Subject-specific estimates of hemodynamic response function from right cerebellum (cerebellar and pontine regions) and left motor cortex (neocortical and diencephalic regions) were used to compute activations. The $Y$ coordinate values depicted below the sections correspond to the Talairach coordinate system (Schmahmann et al., 2000; Talairach \& Tournoux, 1988).

cerebellum and neocortex using a slow event-related fingerresponse task (Fig. $1 \mathrm{~b}$ and c), and convolution of these HRFs with the encoding, maintenance and retrieval event waveforms was used to construct customized regressors for the analyses.

During the encoding phase, opercular portions of the left inferior frontal gyrus were activated along with bilateral precentral gyri, and supplementary motor area. These regions exhibited minimal activations in the ensuing maintenance and retrieval phases. As hypothesized, a similar pattern of activation was observed in the right superior cerebellar hemisphere (VI/Crus I). Activations related to visual processing, including the occipital and fusiform gyri, were also predominately confined to the encoding phase, as were activations in the hippocampus. Although activations in the left inferior parietal and right cerebellar regions began to appear during the encoding period, these regions, in sharp contrast to the frontal and superior cerebellar regions described above, showed continuing increases and reached maximum intensity during the maintenance phase. Activation in the thalamus and pontine brainstem was the greatest during the encoding phase, but pontine activation was also observed to a lesser extent during maintenance, and thalamic activation to a lesser extent during retrieval. Activation in the cingulate gyrus was observed during the encoding phase and remained throughout the maintenance and retrieval phases.

In addition to left inferior parietal and right inferior cerebellar (VIIB/VIIIA) activations, the maintenance phase was 
characterized by recruitment of activation bilaterally in more anterior triangular portions of the inferior frontal gyrus, as well as in middle and orbital frontal gyri. Caudate and putamen activations were observed during maintenance, but did not extend into the retrieval phase. The retrieval phase was largely characterized by increases in the left middle frontal and right orbital frontal gyri, and a considerable reduction of activation in posterior regions of the brain.

Table 1

Activations within telencephalon and diencephalon based on neocortical HRF

\begin{tabular}{|c|c|c|c|c|c|c|c|}
\hline Hemisphere & $X$ & $Y$ & $Z$ & $\operatorname{SPM}\{Z\}$ & $N_{\text {Vox }}$ & Brain structures & BA \\
\hline \multicolumn{8}{|l|}{$\overline{\text { Encoding }}$} \\
\hline Left & -46 & -76 & -11 & 5.4 & 3375 & Inferior occipital gyrus & 18 \\
\hline Left & -48 & -59 & -12 & 5.4 & & Fusiform gyrus & 37 \\
\hline Left & -53 & -71 & -12 & 5.0 & & Fusiform gyrus & 19 \\
\hline Left & -46 & -80 & -4 & 4.9 & & Inferior occipital gyrus & 19 \\
\hline Left & -63 & 8 & 11 & 5.4 & 2128 & Inferior frontal gyrus & 44 \\
\hline Left & -51 & 0 & 44 & 5.4 & & Precentral gyrus & 6 \\
\hline Left & -57 & -11 & 47 & 5.0 & & Precentral gyrus & 4 \\
\hline Left & -51 & -2 & 37 & 4.8 & & Precentral gyrus & 6 \\
\hline Left & -20 & -35 & -2 & 5.4 & 2124 & Parahippocampal gyrus & \\
\hline Left & -26 & -37 & -7 & 5.2 & & Parahippocampal gyrus & \\
\hline Left & -22 & -36 & -13 & 5.1 & & Parahippocampal gyrus & \\
\hline Left & -32 & -30 & -10 & 4.7 & & Hippocampus & \\
\hline Left & -28 & -31 & -2 & 4.5 & & Hippocampus & \\
\hline Left & -6 & 3 & 51 & 4.4 & 1031 & Medial frontal gyrus & 6 \\
\hline Left & -2 & 8 & 46 & 4.3 & & Cingulate gyrus & 32 \\
\hline Left & -10 & 3 & 68 & 4.3 & & Superior frontal gyrus & 6 \\
\hline Left & -2 & 3 & 59 & 4.2 & & Medial frontal gyrus & 32 \\
\hline Right & 24 & -33 & -2 & 5.4 & 2806 & Parahippocampal gyrus & \\
\hline Right & 26 & -33 & -8 & 5.2 & & Parahippocampal gyrus & \\
\hline Right & 18 & -10 & -3 & 4.7 & & Medial globus pallidus & \\
\hline Right & 42 & -7 & 56 & 5.4 & 984 & Precentral gyrus & 4 \\
\hline Right & 55 & -7 & 50 & 5.4 & & Precentral gyrus & 4 \\
\hline Right & 57 & -2 & 42 & 5.0 & & Precentral gyrus & $4 / 6$ \\
\hline Right & 34 & -5 & 63 & 4.9 & & Precentral gyrus & 6 \\
\hline Right & 28 & -99 & 7 & 3.5 & 48 & Middle occipital gyrus & 18 \\
\hline Right & 24 & -98 & 14 & 3.5 & & Middle occipital gyrus & $18 / 19$ \\
\hline Right & 63 & 4 & 5 & 4.0 & 48 & Superior temporal gyrus & 22 \\
\hline \multicolumn{8}{|l|}{ Maintenance } \\
\hline Left & -12 & 4 & 48 & 5.4 & 1124 & Cingulate gyrus & 24 \\
\hline Left & -4 & 6 & 48 & 5.4 & & Cingulate gyrus & 32 \\
\hline Left & -61 & 9 & 20 & 4.8 & 860 & Inferior frontal gyrus & 44 \\
\hline Left & -53 & 6 & 7 & 4.8 & & Inferior frontal gyrus & 44 \\
\hline Left & -55 & 7 & 25 & 4.7 & & Inferior frontal gyrus & 44 \\
\hline Left & -36 & 27 & -10 & 5.0 & 472 & Inferior frontal gyrus & $45 / 47$ \\
\hline Left & -40 & -47 & 41 & 5.1 & 227 & Inferior parietal lobule & 40 \\
\hline Left & -18 & 9 & -6 & 4.0 & 172 & Putamen & \\
\hline Left & -10 & 11 & -7 & 3.9 & & Nucleus accumbens & \\
\hline Left & -48 & -61 & -9 & 4.5 & 94 & Inferior temporal gyrus & 37 \\
\hline Right & 28 & 25 & -6 & 3.9 & 223 & Inferior frontal gyrus & $45 / 47$ \\
\hline Right & 12 & 13 & -6 & 3.5 & 67 & Nucleus accumbens & \\
\hline \multicolumn{8}{|l|}{ Retrieval } \\
\hline Left & -4 & 31 & 28 & 4.8 & 1165 & Cingulate gyrus & 32 \\
\hline Left & -10 & 18 & 43 & 4.3 & & Cingulate gyrus & 32 \\
\hline Left & -6 & 0 & 48 & 4.3 & & Cingulate gyrus & 32 \\
\hline Left & -53 & 9 & 25 & 4.8 & 738 & Inferior frontal gyrus & 44 \\
\hline Left & -61 & 9 & 24 & 4.6 & & Inferior frontal gyrus & 44 \\
\hline Left & -16 & -15 & 6 & 4.4 & 129 & Thalamus, VPLN & \\
\hline Left & -36 & -5 & 61 & 4.7 & 116 & Middle frontal gyrus & 6 \\
\hline Left & -44 & 13 & -6 & 3.6 & 60 & Insula & \\
\hline Right & 50 & 13 & -11 & 4.2 & 418 & Insula/inferior frontal gyrus & $44 / 45$ \\
\hline Right & 44 & 10 & -2 & 4.1 & & Insula & $44 / 45$ \\
\hline Right & 6 & -19 & 18 & 4.5 & 89 & Thalamus & \\
\hline
\end{tabular}

Note: IFG-inferior frontal gyrus; SMA—supplementary motor area. Talairach coordinates of activation maxima in the cerebellum obtained from the atlas of Schmahmann et al. (2000). Z-score values are depicted representing an uncorrected $p$-value threshold of 0.001 . The number of voxels in a cluster of contiguous $2 \mathrm{~mm} \times 2 \mathrm{~mm} \times 2 \mathrm{~mm}$ voxels is depicted. Non-bolded entries represent local maxima within the main cluster. 
Table 2

Activations below diencephalon based on cerebellar HRF

\begin{tabular}{|c|c|c|c|c|c|c|c|}
\hline Hemisphere & $X$ & $Y$ & $Z$ & $\operatorname{SPM}\{Z\}$ & $N_{\text {Vox }}$ & Brain structures & Lobule \\
\hline \multicolumn{8}{|l|}{ Encoding } \\
\hline Left & -18 & -27 & -32 & 4.4 & 232 & Brainstem, pons & \\
\hline Left & -10 & -23 & -32 & 4.4 & & Brainstem, pons & \\
\hline Left & -24 & -33 & -34 & 4.2 & & Brainstem, pons & \\
\hline Right & 34 & -64 & -34 & 5.1 & 1123 & Superior cerebellum & VI/Crus I \\
\hline Right & 46 & -86 & -16 & 4.4 & & Superior posterior cerebellum & Crus I \\
\hline Right & 38 & -56 & -36 & 4.3 & & Superior cerebellum & Crus I \\
\hline Right & 38 & -67 & -29 & 4.2 & & Superior cerebellum & Crus I \\
\hline Right & 28 & -41 & -33 & 4.4 & 301 & Superior cerebellum & $\mathrm{V}$ \\
\hline Right & 0 & -79 & -21 & 4.0 & 149 & Cerebellar vermis & VI \\
\hline Right & 26 & -72 & -49 & 4.1 & 123 & Inferior cerebellum & VIIB/VIIIA \\
\hline \multicolumn{8}{|l|}{ Maintenance } \\
\hline Left & -16 & -24 & -22 & 3.7 & 25 & Brainstem, pons & \\
\hline Right & 18 & -70 & -44 & 4.3 & 149 & Inferior cerebellum & VIIB/VIIIA \\
\hline \multicolumn{8}{|l|}{ Retrieval } \\
\hline Left & -2 & -81 & -23 & 3.93 & 33 & Posterior cerebellar vermis & VI \\
\hline
\end{tabular}

\section{Discussion}

Two main conclusions regarding cerebro-cerebellar network involvement in verbal working memory can be drawn from the results of this investigation. The first is that the superior and inferior cerebellar regions are maximally responsive at different phases of the task, with the superior portion showing the greatest activation during the encoding phase and the inferior region exhibiting the greatest response during the maintenance phase. The second is that predicted patterns of concordant cerebro-cerebellar activation were observed, i.e., left frontal regions in tandem with superior cerebellum and left parietal regions in tandem with right inferior cerebellum.

These findings provide further evidence for cerebrocerebellar involvement in cognition and suggest further refinement of the model postulated by Desmond et al. (1997). Specifically, we propose that the recruitment of the superior cerebellum in the encoding phase of the task reflects this region's involvement with the rapid translation of the consonant sequence into an articulatory trajectory required to initiate the phonological loop. The concomitant encoding-related activation observed in opercular portions of the inferior frontal gyrus and premotor cortex (BA 44,6) suggests that these frontal regions are rapidly recruited along with the superior cerebellum to initiate articulatory rehearsal. More anterior regions of frontal cortex (BA 44, 45, 47) were found to be active throughout the maintenance period - these regions were also found to be active during verbal working memory but not during a rehearsal control task (Chen \& Desmond, 2005). This pattern of results suggests that in the Sternberg task these anterior frontal regions support internally guided aspects of articulatory rehearsal, whereas the posterior frontal regions are more externally driven. Chein and Fiez (2001) similarly noted that the encoding-related activation observed in left dorsal BA
44 suggested that this region may contribute to the first effortful organizational stage of rehearsal, in a manner consistent with the model of Naveh-Benjamin and Jonides (1984). They further noted that (superior) cerebellar activation observed in their study was, contrary to their expectations, observed for the encoding period but did not persist into the maintenance period. The absence of maintenance-related cerebellar activation in that study was likely due to scan coverage that was limited to the superior cerebellum. Our observations are consistent with those of Chein and Fiez, but also revealed maintenance-related cerebellar activation that was confined to the more inferior cerebellar region. These inferior cerebellar activations during the maintenance phase may reflect the continued contribution of the cerebellum in guiding the articulatory trajectory via phonologically based information from the parietal region, as originally proposed by Desmond et al. (1997). However an alternative possibility-one that is perhaps more consistent with recent findings that neocortical and cerebellar regions are reciprocally connected in closed loops (Kelly \& Strick, 2003) —is that parietal and inferior cerebellar activations during this phase comprise a cerebro-cerebellar loop for sustaining the phonological store (see Ravizza, Delgado, Chein, Becker, and Fiez (2004) for alternative accounts of parietal involvement in verbal working memory). In either case one salient distinction between inferior cerebellar and parietal activation is that, although both regions showed the strongest activation during the maintenance phase, $56 \%$ of the inferior cerebellar voxels that were activated during the maintenance phase were also activated during the encoding phase. In contrast, only $6 \%$ of the left parietal voxels exhibited a similar pattern. The presence of encoding-related activation may indicate that the inferior cerebellum is capable of assisting different neocortical regions at different phases of the task. However, the results of Chen and Desmond (2005), in 
which an encoding-intensive non-memory rehearsal control task elicited robust activation in frontal regions and superior cerebellum, but not in the inferior cerebellum (even with liberal $p$-value thresholds), seem inconsistent with this interpretation. An alternative possibility is that phonological storage related processing begins during the initial presentation of letters, i.e., before the actual maintenance phase begins, and that, consistent with its hypothesized role in rapid ballistic movements, the cerebellum engages in this processing more rapidly than does the parietal region.

Additional support for this model can be found from recent neuroimaging results. For example, a functional link between Broca's area and the right superior cerebellum was observed in an investigation of alcoholic and non-alcoholic activation during verbal working memory (Desmond et al., 2003b). Greater activation in the high versus low load comparison was observed for alcoholics compared to non-alcoholic controls only in left BA 44 and right hemispheric cerebellar lobule VI, despite comparable levels of behavioral performance as measured by both accuracy and reaction time. These results may reflect a compensatory increase in this cerebrocerebellar circuit-a circuit that may have been compromised by chronic alcohol consumption-in order to maintain comparable levels of task performance. These findings may extend to other patient populations-for example the close coupling of superior cerebellar and frontal speech areas might explain why speech deficits are more commonly observed in patients after infarctions of the superior cerebellar artery, but not the posterior inferior cerebellar artery (Ackermann, Vogel, Petersen, \& Poremba, 1992). Similarly, anomalies in right anterior cerebellar and inferior frontal regions have been implicated in rapid naming difficulties of dyslexic children (Eckert et al., 2003).

Similar examples of frontal/superior cerebellar and parietal/inferior cerebellar concordance have been demonstrated using manipulations of task requirements. For example, a conjunction of cerebro-cerebellar regions recruited for a rehearsal control task (repeatedly reading and covertly rehearsing either six letters or one letter at a pace designed to mimic the motoric aspects of articulatory rehearsal but without the memory requirement) with regions recruited for verbal working memory revealed common activations in the superior cerebellum and posterior frontal regions, presumably reflecting the common encoding and articulatory requirements of the two tasks (Chen \& Desmond, 2005; Desmond, 2001b). In contrast, inferior cerebellum and inferior parietal regions were recruited only during the verbal working memory task and not the rehearsal control task, demonstrating that the latter regions are invoked when the phonological store is required. This parietal/inferior cerebellar circuit has also been shown to increase activation over extended verbal working memory practice, suggesting that these regions are responsible for practice-related enhancement of verbal working memory efficiency (Kirschen, Chen, Schraedley-Desmond, $\&$ Desmond, 2004, 2005). Such efficiency is manifested behaviorally by significant reaction time reductions over ex- tended practice. These findings further emphasize that the superior and inferior cerebellar activations are manifested under different circumstances during verbal working memory; the independence of these activations is consistent with functionally distinct roles.

The results of the present investigation add to a growing number of investigations of verbal working memory that have used the Sternberg task or similar item recognition tasks to examine issues such as the neural substrates of phonological storage versus articulatory rehearsal (Awh et al., 1996; Fiez et al., 1996; Jonides et al., 1998; Paulesu et al., 1993), verbal versus non-verbal memory (Gruber \& von Cramon, 2001, 2003; Smith \& Jonides, 1997; Smith, Jonides, \& Koeppe, 1996), maintenance versus manipulation of information (D'Esposito, Postle, Ballard, \& Lease, 1999; D’Esposito, Postle, \& Rypma, 2000; Rypma, Prabhakaran, Desmond, Glover, \& Gabrieli, 1999; Smith \& Jonides, 1999; Veltman et al., 2003), interfering versus non-interfering stimuli (Bunge, Ochsner, Desmond, Glover, \& Gabrieli, 2001; Gruber, 2001; Jonides et al., 1998) controlled versus automatic processing (Jansma, Ramsey, Slagter, \& Kahn, 2001), and encoding versus maintenance versus retrieval related responding (Chein \& Fiez, 2001; Manoach, Greve, Lindgren, \& Dale, 2003; Rypma \& D'Esposito, 1999). The locations of verbal working-memory-dependent activations in the present study are consistent with regions found to be active in these and other studies of verbal working memory (see Fiez et al. (1996) for a review) and include premotor cortex (BA 6), inferior frontal regions (BA 44, 45), parietal cortex (BA 40), extrastriate cortex (BA 18, 19), anterior cingulate gyrus (BA 32 ), and cerebellum. However, activation in right dorsolateral prefrontal cortex (BA 9/46) was absent in the present study, in contrast to other studies. For example, Rypma et al. (1999) demonstrated increased right dorsolateral prefrontal activation in the load 6 versus load 1 comparison relative to the load 3 versus load 1 comparison. The present study did observe right frontal activation during the retrieval phase of the task (see Fig. 2, at $y=14$ and 19), but this activation was localized to BA 44,45. Given that more dorsolateral prefrontal regions tend to be engaged for tasks that require executive processing (reviewed by Smith and Jonides (1999)), the absence of BA 9/46 activation in the present study may reflect an insufficient increase in executive processing demands between the load 2 and load 6 conditions. However, an alternative possibility, or contributing factor, may be that the timecourse of dorsolateral prefrontal activations did not conform well to the encoding, maintenance, and retrieval regressors used for analyses (e.g., see Cohen et al., 1997, who reported sustained activation throughout the trial in this region).

Although our model of cerebro-cerebellar function in verbal working memory emphasizes guidance of the articulatory motor trajectory in the phonological loop, elements of the model, as well as the observed patterns of activation, are consistent with other theoretical accounts of cerebellar contributions to cognition. For example, the robust encodingrelated activation that we observed in the superior cerebellum, 
in conjunction with activations observed in the fusiform and left inferior frontal gyri, seems consistent with the hypothesized role of the cerebellum in sensory acquisition, a role that has been emphasized in several other highly sensory-based paradigms (Allen, Buxton, Wong, \& Courchesne, 1997; Gao et al., 1996; Jueptner et al., 1997; Parsons \& Fox, 1997; Sobel et al., 1998) and theoretical accounts of cerebellar function (Bower, 1997). On the other hand, the hypothesized guidance of the articulatory trajectory throughout the maintenance phase, involving the activations observed in the right inferior cerebellum, left inferior parietal lobule, and more anterior portions of the inferior frontal gyrus, is also consistent with cerebellar theories that emphasize processes especially important to motoric control, including error correction (Ito, 1993), predictive feedforward control (Kettner et al., 1997), skill learning (Doyon, Penhune, \& Ungerleider, 2003), coordinated timing of movements (Ivry, 1997), response preparation (Deiber, Ibanez, Sadato, \& Hallett, 1996), and contextresponse linkage (Thach, 1997). It may therefore be the case that strong cerebellar responses to both sensory input and motor preparation could at least partially explain the ubiquity of cerebellar activations reported in neuroimaging investigations of cognition.

It can be seen in Fig. 1 that the least amount of cerebellar activation was observed during the task's retrieval phase, which arguably involves the most manipulation of information and thereby requires the greatest amount of executive function (D'Esposito et al., 1995). The absence of activation during this phase suggests at first glance minimal cerebellar participation in executive function, despite the fact that there are distinct output channels from cerebellar dentate nuclei to dorsolateral prefrontal cortex (via thalamus) from which the cerebellum could significantly influence executive processing (Kelly \& Strick, 2003; Middleton \& Strick, 2000, 2001), and contradicting numerous reports of impaired executive function in cerebellar patients e.g. (Appollonio, Grafman, Schwartz, Massaquoi, \& Hallett, 1993; Botez, Botez, Elie, \& Attig, 1989; Bracke-Tolkmitt et al., 1989; Canavan, Sprengelmeyer, Diener, \& Hoemberg, 1994; Fiez, Petersen, Cheney, \& Raichle, 1992; Levisohn, Cronin-Golomb, \& Schmahmann, 2000; Schmahmann \& Sherman, 1998; Silveri, Leggio, \& Molinari, 1994). However, additional evidence (Chen, Pryor, \& Desmond, 2002) obtained from 14 subjects scanned in a limited field of view restricted to the cerebellum, suggested that the executive requirements for responding to a single probe may not have been sufficient to recruit cerebellar participation. Fig. 3 illustrates that when subjects were given two probes concurrently, and asked to choose if one or both probes matched the encoding stimuli, reaction times were longer relative to the oneprobe condition of the present study; this apparent increase in difficulty was associated with considerably more retrievalrelated cerebellar activation. Additional investigations will be needed to further characterize, in the same group of subjects, the conditions in which increasing executive demand results in increased cerebellar activations.

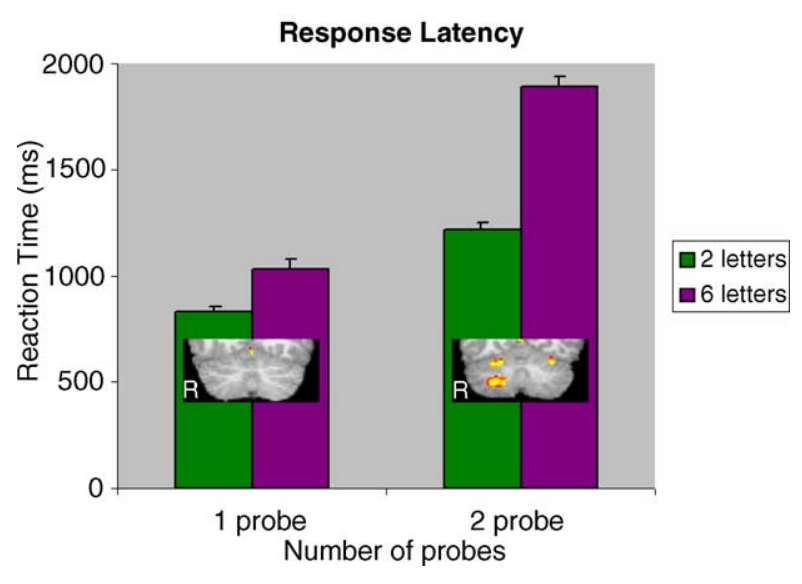

Fig. 3. Increasing the task difficulty during the retrieval phase leads to greater retrieval-related cerebellar activation. Graphs illustrate significant increases $(F(1,27)=50.86, p<.00001)$ in reaction time for correct responses when subjects are required to respond to two simultaneously presented probes instead of just one. Greater retrieval-phase-related cerebellar activation $(p<.001)$ is also observed under the two-probe conditions, reflecting possible cerebellar participation in executive processes.

In summary, the results of the present study further support cerebellar involvement in verbal working memory and are consistent with patient (Silveri, Di Betta, Filippini, Leggio, \& Molinari, 1998) and transcranial magnetic stimulation (Desmond, Chen, Shieh, \& Boxer, 2003a) results indicating that cerebellar disruption adversely affects verbal working memory performance. The present study further suggests that two functionally distinct cerebro-cerebellar networks participate in verbal working memory. These networks are recruited during different phases of the task and are postulated to support the changing cognitive requirements that evolve during the timecourse of the task.

\section{Acknowledgements}

The authors gratefully acknowledge Dr. Gary Glover for providing consultation and support for the pulse sequences used for fMRI, and Jenea Boshart for subject recruitment and project coordination. Supported by NIMH (MH60234).

\section{References}

Ackermann, H., Vogel, M., Petersen, D., \& Poremba, M. (1992). Speech deficits in ischaemic cerebellar lesions. Journal of Neurology, 239(4), 223-227.

Allen, G., Buxton, R. B., Wong, E. C., \& Courchesne, E. (1997). Attentional activation of the cerebellum independent of motor involvement. Science, 275(5308), 1940-1943.

Appollonio, I. M., Grafman, J., Schwartz, V., Massaquoi, S., \& Hallett, M. (1993). Memory in patients with cerebellar degeneration. Neurology, 43(8), 1536-1544.

Awh, E., Jonides, J., Smith, E. E., Schumacher, E. H., Koeppe, R. A., \& Katz, S. (1996). Dissociation of storage and rehearsal in verbal working memory: Evidence from positron emission tomography. Psychological Science, 7(1), 25-31. 
Baddeley, A. (1992). Working memory. Science, 255(5044), 556-559.

Baddeley, A. D. (1986). Working memory. Oxford: Oxford University Press.

Botez, M. I., Botez, T., Elie, R., \& Attig, E. (1989). Role of the cerebellum in complex human behavior. Italian Journal of the Neurological Sciences, 10(3), 291-300.

Bower, J. M. (1997). Control of sensory data acquisition. International Review of Neurobiology, 41, 489-513.

Bracke-Tolkmitt, R., Linden, A., Canavan, A. G. M., Rockstroh, B., Scholz, E., Wessel, K., et al. (1989). The cerebellum contributes to mental skills. Behavioral Neuroscience, 103(2), 442-446.

Brodal, P. (1979). The pontocerebellar projection in the rhesus monkey: An experimental study with retrograde axonal transport of horseradish peroxidase. Neuroscience, 4(2), 193-208.

Bunge, S. A., Ochsner, K. N., Desmond, J. E., Glover, G. H., \& Gabrieli, J. D. (2001). Prefrontal regions involved in keeping information in and out of mind. Brain, 124(Pt 10), 2074-2086.

Canavan, A. G. M., Sprengelmeyer, R., Diener, H. C., \& Hoemberg, V. (1994). Conditional associative learning is impaired in cerebellar disease in humans. Behavioral Neuroscience, 108(3), 475-485.

Chein, J. M., \& Fiez, J. A. (2001). Dissociation of verbal working memory system components using a delayed serial recall task. Cerebral Cortex, 11(11), 1003-1014.

Chen, S. A., Pryor, M. R., \& Desmond, J. E. (2002). Cerebellar involvement in encoding, maintenance, and retrieval components of verbal working memory: An event-related fmri study. Society for Neuroscience Abstract Viewer and Itinerary Planner, Abstract No. 416.414.

Chen, S. H. A., \& Desmond, J. E. (2005). Cerebrocerebellar networks during articulatory rehearsal and verbal working memory tasks. Neuroimage, 24, 332-338.

Cohen, J. D., MacWhinney, B., Flatt, M., \& Provost, J. (1993). PsyScope: A new graphic interactive environment for designing psychology experiments. Behavioral Research Methods, Instruments, and Computers, 25, 257-271

Cohen, J. D., Perlstein, W. M., Braver, T. S., Nystrom, L. E., Noll, D. C., Jonides, J., \& Smith, E. E. (1997). Temporal dynamics of brain activation during a working memory task. Nature, 386(6625), 604-608.

Deiber, M. P., Ibanez, V., Sadato, N., \& Hallett, M. (1996). Cerebral structures participating in motor preparation in humans: A positron emission tomography study. Journal of Neurophysiology, 75(1), 233-247.

Desmond, J. E. (2001a). Cerebellar involvement in cognitive function: Evidence from neuroimaging. International Review of Psychiatry, 13, 283-294.

Desmond, J. E. (2001b). Cerebro-cerebellar interactions in articulatory loops with and without working memory: An fMRI study. Society for Neuroscience Abstracts, 27, 219.

Desmond, J. E., Chen, S. A., Shieh, P. B., \& Boxer, A. L. (2003). Right cerebellar single-pulse transcranial magnetic stimulation (tms) affects verbal working memory performance. Society for Neuroscience Abstract Viewer and Itinerary Planner, Abstract No. 287.288.

Desmond, J. E., Chen, S. H., DeRosa, E., Pryor, M. R., Pfefferbaum, A., \& Sullivan, E. V. (2003). Increased frontocerebellar activation in alcoholics during verbal working memory: An fMRI study. Neuroimage, 19(4), 1510-1520.

Desmond, J. E., \& Fiez, J. A. (1998). Neuroimaging studies of the cerebellum: Language, learning and memory. Trends in Cognitive Sciences, 2(9), 355-362.

Desmond, J. E., Gabrieli, J. D., Wagner, A. D., Ginier, B. L., \& Glover, G. H. (1997). Lobular patterns of cerebellar activation in verbal workingmemory and finger-tapping tasks as revealed by functional MRI. Journal of Neuroscience, 17(24), 9675-9685.

Desmond, J. E., \& Lim, K. O. (1997). On- and offline Talairach registration for structural and functional MRI studies. Human Brain Mapping, $5(1), 58-73$.

D’Esposito, M., Detre, J. A., Alsop, D. C., Shin, R. K., Atlas, S., \& Grossman, M. (1995). The neural basis of the central executive system of working memory. Nature, 378(6554), 279-281.
D’Esposito, M., Postle, B. R., Ballard, D., \& Lease, J. (1999). Maintenance versus manipulation of information held in working memory: An event-related fMRI study. Brain Cognition, 41(1), 66-86.

D’Esposito, M., Postle, B. R., \& Rypma, B. (2000). Prefrontal cortical contributions to working memory: Evidence from event-related fMRI studies. Experimental Brain Research, 133(1), 3-11.

Doyon, J., Penhune, V., \& Ungerleider, L. G. (2003). Distinct contribution of the cortico-striatal and cortico-cerebellar systems to motor skill learning. Neuropsychologia, 41(3), 252-262.

Eckert, M. A., Leonard, C. M., Richards, T. L., Aylward, E. H., Thomson, J., \& Berninger, V. W. (2003). Anatomical correlates of dyslexia: Frontal and cerebellar findings. Brain, 126(Pt 2), 482-494.

Fiez, J. A., Petersen, S. E., Cheney, M. K., \& Raichle, M. E. (1992). Impaired non-motor learning and error detection associated with cerebellar damage. A single case study. Brain, 115, 155-178.

Fiez, J. A., \& Raichle, M. E. (1997). Linguistic processing. In J. Schmahmann (Ed.), The cerebellum and cognition international review of neurobiology: vol. 41 (pp. 233-254). San Diego, CA: Academic Press.

Fiez, J. A., Raife, E. A., Balota, D. A., Schwarz, J. P., Raichle, M. E., \& Petersen, S. E. (1996). A positron emission tomography study of the short-term maintenance of verbal information. Journal of Neuroscience, 16(2), 808-822.

Gao, J. H., Parsons, L. M., Bower, J. M., Xiong, J., Li, J., \& Fox, P. T. (1996). Cerebellum implicated in sensory acquisition and discrimination rather than motor control. Science, 272(5261), 545547.

Glover, G. H., \& Law, C. S. (2001). Spiral-in/out BOLD fMRI for increased SNR and reduced susceptibility artifacts. Magnetic Resonance in Medicine, 46(3), 515-522.

Gruber, O. (2001). Effects of domain-specific interference on brain activation associated with verbal working memory task performance. Cerebral Cortex, 11(11), 1047-1055.

Gruber, O., \& von Cramon, D. Y. (2001). Domain-specific distribution of working memory processes along human prefrontal and parietal cortices: A functional magnetic resonance imaging study. Neuroscience Letters, 297(1), 29-32.

Gruber, O., \& von Cramon, D. Y. (2003). The functional neuroanatomy of human working memory revisited Evidence from 3-T fMRI studies using classical domain-specific interference tasks. Neuroimage, 19(3), 797-809.

Ito, M. (1993). Synaptic plasticity in the cerebellar cortex and its role in motor learning. Canadian Journal of the Neurological Sciences, 20 Suppl, 3, S70-S74.

Ivry, R. (1997). Cerebellar timing systems. International Review of Neurobiology, 41, 555-573.

Jansma, J. M., Ramsey, N. F., Slagter, H. A., \& Kahn, R. S. (2001). Functional anatomical correlates of controlled and automatic processing. Journal of Cognitive Neuroscience, 13(6), 730-743.

Jonides, J., Schumacher, E. H., Smith, E. E., Koeppe, R. A., Awh, E., Reuter-Lorenz, P. A., et al. (1998). The role of parietal cortex in verbal working memory. Journal of Neuroscience, 18(13), 5026-5034.

Jueptner, M., Ottinger, S., Fellows, S. J., Adamschewski, J., Flerich, L., Muller, S. P., et al. (1997). The relevance of sensory input for the cerebellar control of movements. Neuroimage, 5(1), 41-48.

Kelly, R. M., \& Strick, P. L. (2003). Cerebellar loops with motor cortex and prefrontal cortex of a nonhuman primate. Journal of Neuroscience, 23(23), 8432-8444.

Kettner, R. E., Mahamud, S., Leung, H. C., Sitkoff, N., Houk, J. C., Peterson, B. W., et al. (1997). Prediction of complex two-dimensional trajectories by a cerebellar model of smooth pursuit eye movement. Journal of Neurophysiology, 77(4), 2115-2130.

Kirschen, M. P., Chen, S. H. A., Schraedley-Desmond, P., \& Desmond, J. E. (2004). Increases in cerebro-cerebellar activation with increasing memory load and task practice: An fMRI study. International Society for Magnetic Resonance in Medicine, Abstracts.

Kirschen, M. P., Chen, S. H. A., Schraedley-Desmond, P., \& Desmond, J. E. (2005). Load- and practice-dependent increases in cerebro- 
cerebellar activation in verbal working memory: An fMRI study. Neuroimage, 24, 462-472.

Levisohn, L., Cronin-Golomb, A., \& Schmahmann, J. D. (2000). Neuropsychological consequences of cerebellar tumour resection in children: Cerebellar cognitive affective syndrome in a paediatric population. Brain, 123(Pt 5), 1041-1050.

Malm, J., Kristensen, B., Karlsson, T., Carlberg, B., Fagerlund, M., \& Olsson, T. (1998). Cognitive impairment in young adults with infratentorial infarcts. Neurology, 51(2), 433-440.

Manoach, D. S., Greve, D. N., Lindgren, K. A., \& Dale, A. M. (2003). Identifying regional activity associated with temporally separated components of working memory using event-related functional MRI Neuroimage, 20(3), 1670-1684.

Marien, P., Engelborghs, S., Fabbro, F., \& De Deyn, P. P. (2001). The lateralized linguistic cerebellum: A review and a new hypothesis. Brain Language, 79(3), 580-600.

Middleton, F. A., \& Strick, P. L. (2000). Basal ganglia and cerebellar loops: Motor and cognitive circuits. Brain Research Reviews, 31(2-3), 236-250.

Middleton, F. A., \& Strick, P. L. (2001). Cerebellar projections to the prefrontal cortex of the primate. Journal of Neuroscience, 21(2), 700712 .

Naveh-Benjamin, M., \& Jonides, J. (1984). Maintenance rehearsal: A two-component analysis. Journal of Experimental Psychology: Learning, Memory, \& Cognition, 10(3), 369-385., http://www.apa.org/ journals/xlm.html.

Parsons, L. M., \& Fox, P. T. (1997). Sensory and cognitive functions. International Review of Neurobiology, 41, 255-271.

Paulesu, E., Frith, C. D., \& Frackowiak, R. S. (1993). The neural correlates of the verbal component of working memory. Nature, 362(6418), 342-345.

Petersen, S. E., Fox, P. T., Posner, M. I., \& Mintun, M. (1989). Positron emission tomographic studies of the processing of single words. Journal of Cognitive Neuroscience, 1(2), 153-170.

Ravizza, S. M., Delgado, M. R., Chein, J. M., Becker, J. T., \& Fiez, J. A. (2004). Functional dissociations within the inferior parietal cortex in verbal working memory. Neuroimage, 22(2), 562-573.

Rypma, B., \& D'Esposito, M. (1999). The roles of prefrontal brain regions in components of working memory: Effects of memory load and individual differences. Proceedings of the National Academy of Sciences of the United States of America, 96(11), 6558-6563.

Rypma, B., Prabhakaran, V., Desmond, J. E., Glover, G. H., \& Gabrieli, J. D. (1999). Load-dependent roles of frontal brain regions in the maintenance of working memory. Neuroimage, 9(2), 216-226.

Schmahmann, J. D. (1996). From movement to thought: Anatomic substrates of the cerebellar contribution to cognitive processing. Human Brain Mapping, 4, 174-198.
Schmahmann, J. D., Doyon, J., Toga, A. W., Petrides, M., \& Evans, A. C. (2000). MRI atlas of the human cerebellum. San Diego, CA: Academic Press.

Schmahmann, J. D., \& Sherman, J. C. (1998). The cerebellar cognitive affective syndrome. Brain, 121(Pt 4), 561-579.

Scott, R. B., Stoodley, C. J., Anslow, P., Paul, C., Stein, J. F., Sugden, E. M., et al. (2001). Lateralized cognitive deficits in children following cerebellar lesions. Developmental Medicine and Child Neurology, 43(10), 685-691.

Silveri, M. C., Di Betta, A. M., Filippini, V., Leggio, M. G., \& Molinari, M. (1998). Verbal short-term store-rehearsal system and the cerebellum. Evidence from a patient with a right cerebellar lesion. Brain, 121(Pt 11), 2175-2187.

Silveri, M. C., Leggio, M. G., \& Molinari, M. (1994). The cerebellum contributes to linguistic production: A case of agrammatic speech following a right cerebellar lesion [see comments]. Neurology, 44(11), 2047-2050.

Smith, E. E., \& Jonides, J. (1997). Working memory: A view from neuroimaging. Cognitive Psychology, 33(1), 5-42.

Smith, E. E., \& Jonides, J. (1998). Neuroimaging analyses of human working memory. Proceedings of the National Academy of Sciences of the United States of America, 95(20), 12061-12068.

Smith, E. E., \& Jonides, J. (1999). Storage and executive processes in the frontal lobes. Science, 283(5408), 1657-1661.

Smith, E. E., Jonides, J., \& Koeppe, R. A. (1996). Dissociating verbal and spatial working memory using pet. Cerebral Cortex, 6(1), 11-20.

Smith, E. E., Jonides, J., Marshuetz, C., \& Koeppe, R. A. (1998). Components of verbal working memory: Evidence from neuroimaging. Proceedings of the National Academy of Sciences of the United States of America, 95(3), 876-882.

Sobel, N., Prabhakaran, V., Hartley, C. A., Desmond, J. E., Zhao, Z., Glover, G. H., et al. (1998). Odorant-induced and sniff-induced activation in the cerebellum of the human. Journal of Neuroscience, 18(21), 8990-9001.

Sternberg, S. (1966). High-speed scanning in human memory. Science, 153(3736), 652-654.

Talairach, J., \& Tournoux, P. (1988). Co-planar stereotactic atlas of the human brain. Stuttgart: Beorg Thieme Verlag.

Thach, W. T. (1997). Context-response linkage. International Review of Neurobiology, 41, 599-611.

Veltman, D. J., Rombouts, S. A., \& Dolan, R. J. (2003). Maintenance versus manipulation in verbal working memory revisited: An fMRI study. Neuroimage, 18(2), 247-256.

Wildgruber, D., Ackermann, H., \& Grodd, W. (2001). Differential contributions of motor cortex, basal ganglia, and cerebellum to speech motor control: Effects of syllable repetition rate evaluated by fMRI. Neuroimage, 13(1), 101-109. 